
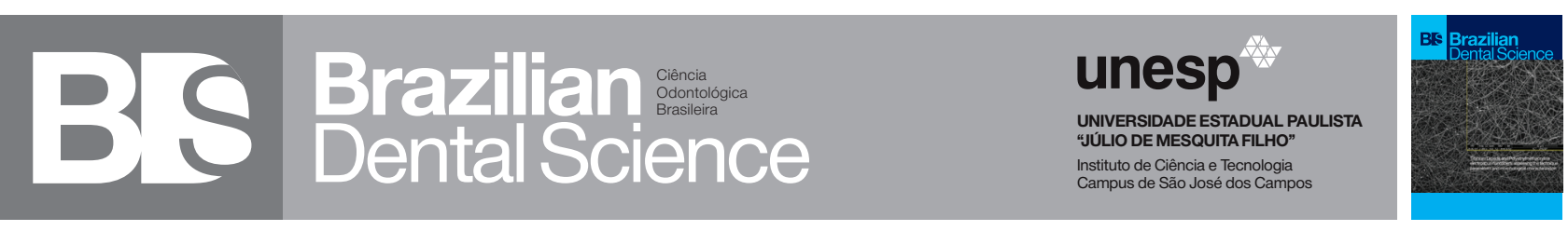

\title{
Minimally invasive aesthetic rehabilitation in composite resin: report of two clinical cases
}

\author{
Reabilitação estética minimamente invasiva em resina composta: relato de dois casos clínicos
}

Priscila Regis PEDREIRA ${ }^{1}$, Janaina Emanuela DAMASCENO ${ }^{1}$, Josué Junior Araújo PIEROTE ${ }^{1}$, Diogo DRESSANO ${ }^{1}$, Giselle Maria MARCHI $^{1}$

1 - Department of Restorative Dentistry - Piracicaba Dental School - State University of Campinas - Piracicaba - SP - Brazil.

\begin{abstract}
Patient demand looking for the perfect smile has been steadily growing in the last few years. Thus, these two case reports discuss some ultra-conservatives approach to achieve good esthetic results. In the first case the clinical protocol of a restorative approach is described, the cosmetic remodeling of the teeth and in the second case, the replacement of extensive unsatisfactory composite restorations. The treatment planning was based on diagnostic waxing and the making of mock-up in both cases. Teeth bleaching were also performed before restoration. To conclude, these conservative relative therapies with composite resin restorations provide an ultra-minimally invasive protocol with excellent esthetic appearances and fuction, especially for young people.
\end{abstract}

\section{KEYWORDS}

Dental esthetics; Composite resins; Smiling; Restoration.

\section{INTRODUCTION}

$\mathrm{P}$ atient demand looking for the perfect 1 smile has been steadily growing in the last few years $[1,2,3]$. The improvement of the adhesive dentistry has allow adding composite resin material to sound tooth surfaces for solve esthetics problems without any preparation, following the minimally invasive principles [3].

The aesthetics of the anterior teeth is the biggest complaint of patients who seek dental office. Usually, the anterior teeth are affected by malformation, anatomic and color alteration,

\section{RESUMO}

A demanda do paciente que procura o sorriso perfeito tem crescido constantemente nos últimos anos. Assim, esses dois relatos de casos clínicos discutem uma abordagem ultraconservadora para alcançar bons resultados estéticos. No primeiro caso é descrito o protocolo clínico de uma abordagem restauradora, a remodelação cosmética dos dentes e, no segundo caso, a substituição de restaurações compostas extensas insatisfatórias. O planejamento do tratamento baseouse em enceramento diagnóstico e a confecção de mock-up. O clareamento dos dentes foi realizado antes da restauração. Para concluir, essas terapias relativas conservadoras com restaurações de resina composta fornecem um protocolo ultra minimamenteinvasivo com excelentes aparências estéticas.

\section{PALAVRAS-CHAVE}

Estética dentária; Resinas compostas; Sorriso; Restauração. hypoplastic defects and less for caries since posterior teeth are more affected than anterior teeth [2]. A multidisciplinary treatment is an interesting approach to improve dental esthetic, the restorative treatment can be combined with periodontal surgery and orthodontic treatments [1].

Restoring anterior teeth requires a carefully attention for the upper central incisors that must be with attractive proportions and dominant since they are the key determinants in evaluating anterior esthetics. There are several treatments options to restore anterior teeth, 
composite resins or ceramics veneers are some of them. Although, ceramic veneers have biological compatibility, color stability and resistance to compression and abrasion, they have high cost and preparation of healthy tooth structure most of the time as disadvantages. Therefore, following the minimal invasive fundamental the direct composite resin is still the first choice for anterior and posterior restorations $[2,4]$.

From the early 1960's, composite resin has been available as an aesthetic material for restorative dentistry and since then there has been a continuous evolution of composite resins, adhesive systems and restorative techniques that have contributed to a significant improvement in aesthetic dentistry [4].

Nowadays, restorations with direct composite resin are successfully recommended in the minimally invasive treatment of all types of black classifications [5]. Moreover, the developments of adhesive techniques have increased the use of conservative restoration options for restore the esthetic appearance of the dentition, including restorations repairs, direct composite resin crowns for anterior and posterior teeth, and direct composite resin reconstruction, usually done in the anterior region for esthetics reasons [5].

Thus, the aim of these two clinical reports is to describe minimally invasive techniques to restore aesthetics of two patients with anterior composite resin restorations.

\section{CASES REPORT}

\section{Case 1}

A 25-year-old female patient who complained about her smile, shape and color of her anterior teeth was referred to the restorative dentistry specialization course at Piracicaba Dental School (University of Campinas, Piracicaba, São Paulo, Brazil). The patient's history showed previous orthodontic treatment, orthodontic surgery, no extractions and no existing restorations. After clinical examination and esthetic analysis was noticed a healthful dentition and a beautiful smile, with the exception of the canines with a sharp aspect, giving her smile a more aggressive appearance, masculine characteristics and the distal incisal angle of both lateral incisors with a little diastema (Figure 1a-d).

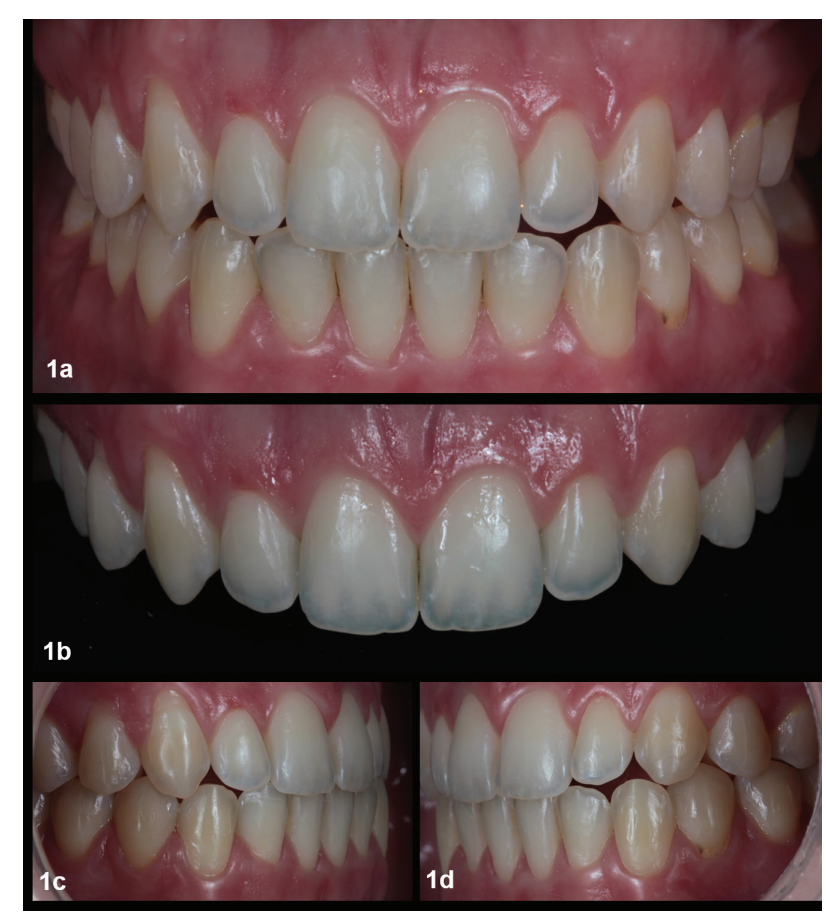

Figure 1: Pretreatment aspect of the intraoral view in maximum intercuspidation (a); maxillary anterior teeth (b); lateral views in maximum intercuspidation ( $c$ and $d$ ).

Models and photographs were prepared for case study. Additionally, a diagnostic waxup was done on the maxillary arch model.

Treatment was initiated with dental office bleaching, three sessions of 15 minutes each were performed three times, with 7-day interval (Whiteness HP 35\%, FGM, Brazil). Afterwards a mock up was conducted using the silicone guide obtained from the wax up diagnostic (figure 1e) and a bis-acrylic resin (Yprov Bisacryl A2, Yller, Brazil). Thereafter some adjustments and tests were performed.

Therefore, the patient was informed about treatment options, and chose the direct composite resin restorations (units: 13, 12, 22 and 23), due to low cost and no teeth preparations need. 
After bleaching treatment was concluded, two weeks were waited to the color to stabilize, then prophylaxis in the restoration region was done with a nylon brush with prophylactic paste and pumice stone with water, followed by color selection. An impression of the mock up was made using a polyvinyl siloxane material (Scan Putty, Yller, Brazil) for obtaining the palatal silicone barrier to guide the making of the resin restorations.

In order to begin the restoration, adequate isolation was performed using a modificated rubber dam isolation (15-25) (Figure 2a) The picture was better adjusted for the rubber dam appears. Then, a Polytetrafluoroethylene (Isotape, TDV, Santa Catarina, Brazil) tape was placed on the adjacent teeth to isolate (Figure 2b). Therefore, enamel etching was performed with 37\% phosphoric acid gel (Condac 37\%, FGM, Brazil) for 30s (Figure 2c), rinsed for 30s and the excess water was removed with air jet. Two layers of the universal adhesive (Single Bond Universal, 3M ESPE, SP, Brazil) was actively applied for 20s each (Figure 2d), and dried gently with a distant air jet for adequate solvent evaporation prior to light curing for 20 s, which was conducted using a light-emitting diode-based light-curing unit done (Radii Cal $1200 \mathrm{~mW} / \mathrm{cm}^{2}$, SDI).

The silicone barrier was seated to insure proper fit of the palatal portion of the teeth, followed by the application of a thin layer of a high translucent resin (WE, Filtek Z350 XT®, 3M ESPE, Brazil) (Figure 2e) onto the barrier. Every resin increment was light-cured for 20s. A second increment with the enamel A1 resin (Estelite Sigma Quite, Tokuyama Dental, Tokyo, Japan) was done with the auxiliary of a polyester strip to reproduce the mesial and distal. Moreover, a final layer with the same resin was applied in the middle third to incisal of the teeth in order to reproduce the enamel. For the canines the aim was to give a more rounded aspect, which characterizes a more feminine smile and for the laterals to reduce the black space.
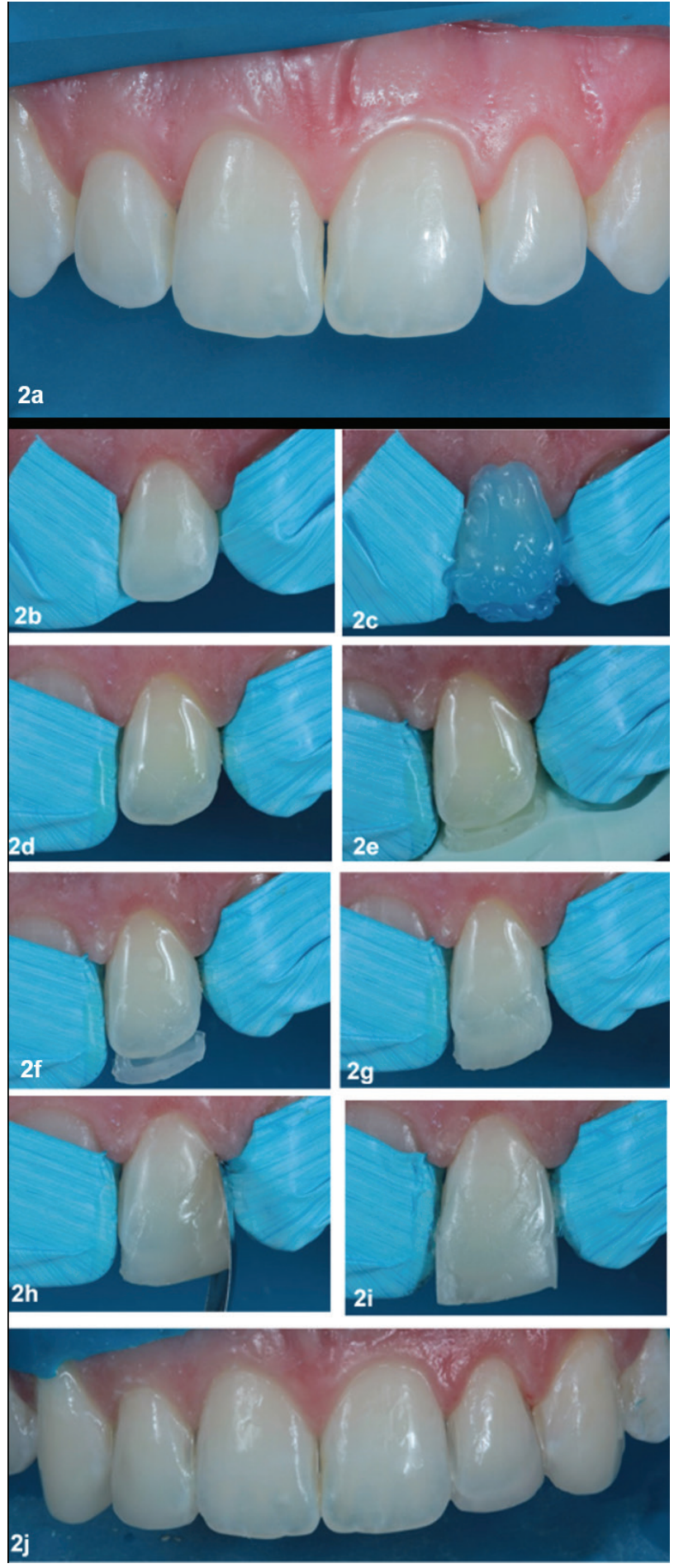

Figure 2: Rubber dam isolation modificated - was adjust (1525) (a); Polytetrafluoroethylene tape to isolate adjacent teeth (b); etched with $37 \%$ phosphoric acid gel (c); apply the universal adhesive (d); first layer of translucid resin (e and f); proximal layer of translucid resin ( $\mathrm{g}$ and $\mathrm{h}$ ); layer of enamel resin (i); final aspect of the resin before polished (j); 
Finally, the rubber dam was removed and diamond burs (KG Sorensen Indústria e Comércio Ltda, Barueri, SP, Brazil) and a flexible abrasive disk (Sof-lex XT, 3M ESPE, Brazil), was used to remove the main excess and improve the macro-anatomy. Laterality and protrusion movements were took into account to perform occlusal adjustment. On a subsequent session, finishing process was done with a series of finishing burs (KG Sorensen Indústria e Comércio Ltda, Barueri, SP, Brazil) and flexible disks (Sof-Lex ${ }^{\mathrm{TM}}$ 3M ESPE, Brazil) in decreasing grains to give the texture and microanatomy. Then, finishing strips (Sof-Lex ${ }^{\mathrm{TM}}, 3 \mathrm{M}$ ESPE, Brazil) were used for the interproximal area to remove any irregularities. Finally, final polishing were performed with diamond polishing paste (Prisma Gloss, Dentsply Caulk, USA) in association to felt disks (Diamond Flex, FGM, Brazil).

After 7 days the patient was recalled to evaluate the definitive esthetic quality of the restoration and rechecking occlusion (Figure 3a-e). Follow up of 18 months was done and treatment successfulness (Figure 4a-c) was confirmed, meeting patients and professionals' expectations.

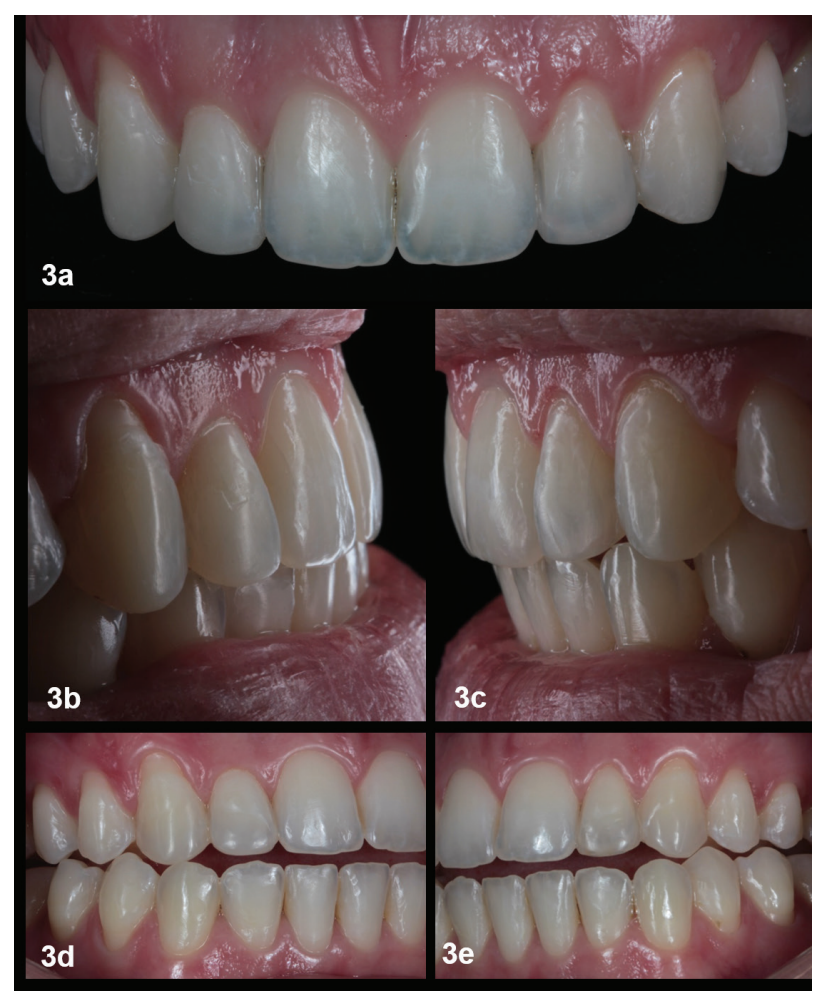

Figure 3: Intraoral view of the final aspect after polish (a), lateral views ( $b$ and $c$ ) and lateral guidence ( $d$ and $e$ ).

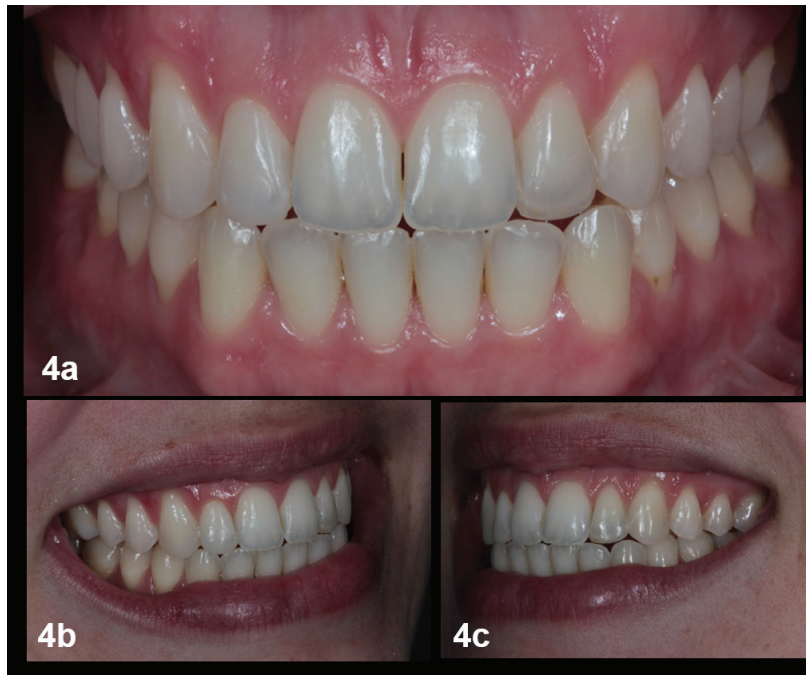

Figure 4: Intraoral view (a) and lateral views (b and $c$ ) in maximum intercuspidation of Follow up after 18 months.

\section{Case 2}

A 20-year-old female patient having smile dissatisfaction about the shape and color of her anterior teeth, presented at the Piracicaba Dental School (University of Campinas, Piracicaba, São Paulo, Brazil) (Figure 5a). The patient's history revealed resin restorations on anterior teeth due to trauma nine years before, previous orthodontic treatment and no extractions. The intra oral examination revealed unsatisfactory resin in the maxillary both central incisors and left lateral with color alteration and overcontoured, inverted smile, gingivitis and no caries (Figure 5b-d). The radiographic examination and pulp vitality showed that the patient's teeth were normal, with no signs of periapical and periodontal injuries.

A diagnostic wax up was done on the maxillary arch model obtained by a plaster model by impression using an alginate material (Hydrogum 5; Zhermack, Badia Polesine, RO, ITALY). The various treatment options were discussed and after considering advantages and limitations, she chose the direct composite resin.

Initial prophylaxis and supra-gingival scaling of calculi were indicated prior to the restoration's procedure. Dental bleaching was performed with three sessions of 15 minutes 
each of in office bleaching three times, with 7-day interval (Whiteness HP 35\%, FGM, Brazil) (Figure 6a).

The final color was selected after two weeks for the color stabilization. Then, prophylaxis and local anesthesia with $2 \%$ lidocaine with 1:80 000 epinephrine (Xylestesin- A; 3M ESPE, Brazil). The existing restorations were removed with a spherical diamond bur \#1014 (KG Sorensen, Barueri, Brazil), used under constant water irrigation (Figure 6b). Then, adequate isolation was performed using a rubber dam isolation modificated (15-25) (Figure 6c). After the teeth preparation, the same adhesive protocol and materials applied in case 1 were performed. The silicone barrier was obtained by an impression of the wax up using a polyvinyl siloxane material (Scan Putty, Yller) and was positioned to reproduce the palatal portion of the teeth with a thin layer of a high translucent nanocomposite (WE, Filtek Z350 XT®, 3M ESPE, Brazil). The appropriate thickness of different composite layers is fundamental to achieve a natural appearance by the restoration. An A1B dentin shade resin (Filtek Z350 XT®, 3M ESPE, Brazil) was used to reproduce the dentin and the mamelons. In addition to that, a final layer with an A1 enamel shade resin (Estelite Sigma Quite, Tokuyama Dental, Tokyo, Japan) was applied on the facial surface. Each resin increment was polymerized for 20 s as close as possible to the material without touching the resin. Finally, aspect of the restorations before polish could be visualized (Figure 5d).

Clinical protocols and materials used for the finishing and polishing were implemented as in case 1 .

After 7 days the patient was recalled to evaluate the definitive esthetic quality of the restoration (Figure 7a-c), rechecking anterior guide (Figure 8a). Follow up of 18 months was done and treatment successfulness (Figure 8b) was confirmed, meeting patients and professionals' expectations.

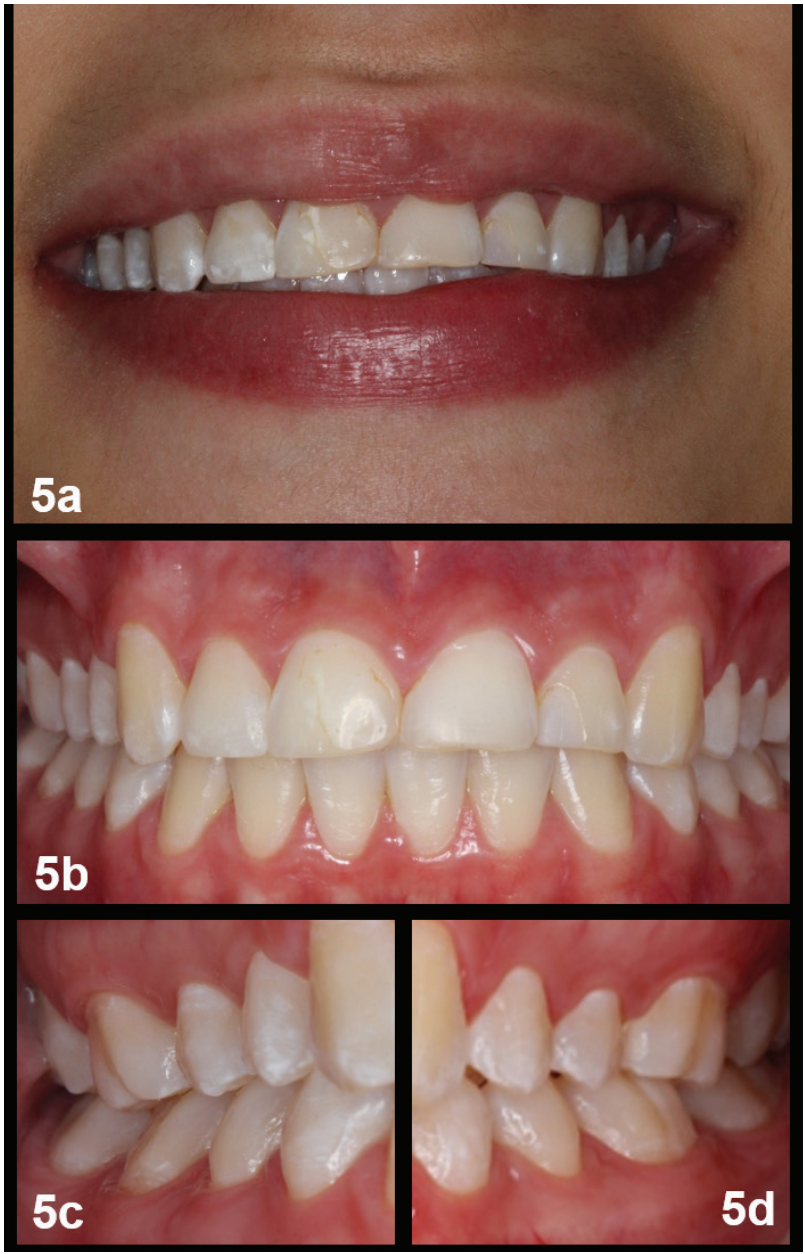

Figure 5: Pretreatment aspect of the extraoral view (a) and intraoral view (b) in maximum intercuspidation; lateral views in maximum intercuspidation (c and d).

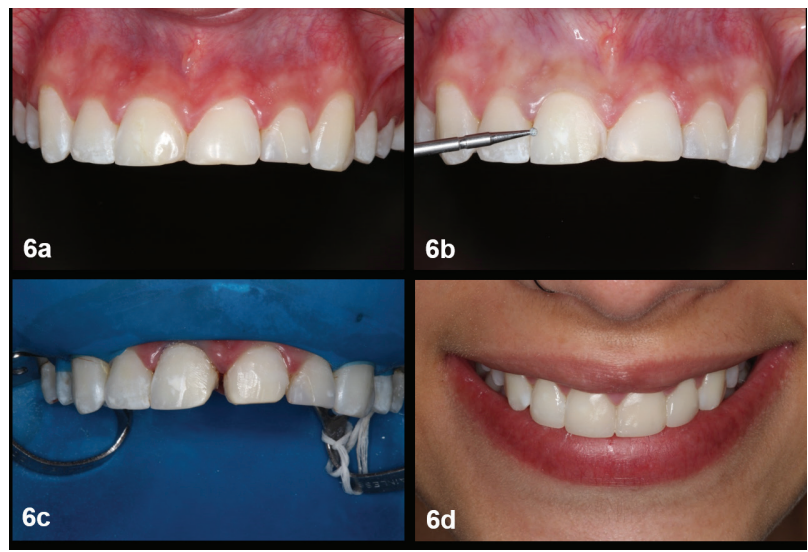

Figure 6: Maxillary anterior teeth after dental bleaching (a); Removing the existing restoration with diamond bur (b); Rubber dam isolation modificated (c); Aspect of the restorations before polish (d). 


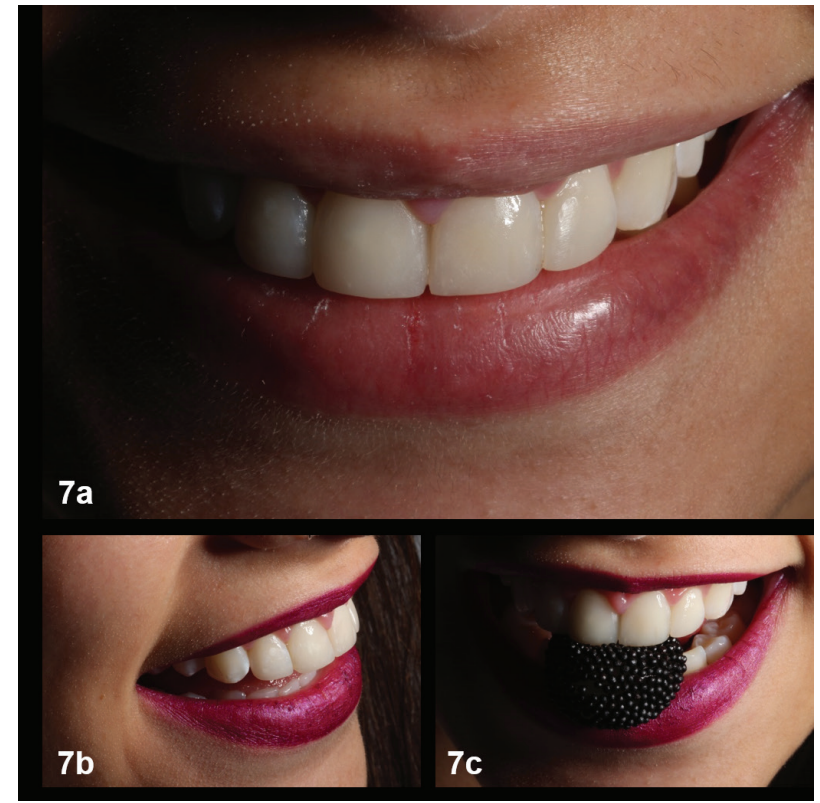

Figure 7: Artistic photos of the final result (a-c).

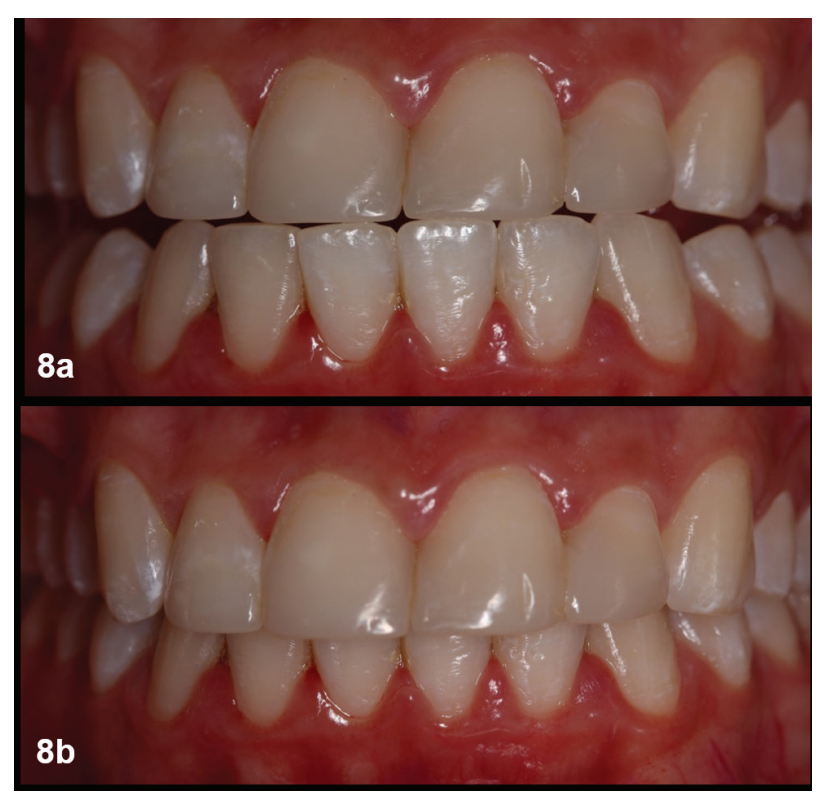

Figure 8: Anterior guide (a) and Intraoral view (b) in maximum intercuspidation of follow up after 18 months.

\section{DISCUSSION}

Preoperative esthetic analysis, including the use of photographs and plaster models, are key factors in the treatment planning [6]. Besides that, the success of any procedure depends on the patient's choice of treatment since it involves their psychological, physical and financial aspects [1].

In case 1 a conservative approach for the management of unaesthetic anterior teeth was illustrated. The resin build-ups generally are performed on sound enamel surface, since no preparation is needed [7]. So, it is well known that enamel bonding to the composite resin have proven effectiveness and the major problem is bonding to dentin. For optimizing bonding is an advisable strategy to do selective enamel etching prior to the application of a mild universal adhesive since it improves the bond strength to enamel and less discoloration at the restorative margins was observed to restorations that involved other conditioning systems $[8,9]$.

In addition, due to the low configuration factor of the restorations, less polymerization shrinkage happened, resulting in fewer cases of microleakage failures [10]. The durability rate of anterior composite resin buildups is nearly $85 \%$ after 5 years, which means that these restorations have a very good long-term prognosis [10].

In case 2 , it was presented the replacement of extensive restorations presenting overcontoured and color alteration. Color mismatch and marginal staining are the major problems that affect the anterior teeth and are easily noticed by patients, since they are in esthetic area. For dentists, restorations in esthetic area is always a challenge, due to the skills needed to mimic the natural tooth and achieving patient's high expectations [11].

Moreover, the combination of bleaching and direct composite resin approach were proposed in both cases reports and have shown to be highly recommended for young adults treatment since it allows a satisfactory, predictable and minimally invasive clinical procedure. Some advantages of the resin are adhesive properties, minimum size of preparation or no preparation preserving sound dental structure, the reinforcement of remaining structure, good clinical durability and aesthetic appearance of matching the natural tooth color. In addition, composite resin restorations 
allow other future treatment options in case of restoration failure, as for example the repair that is done intraoral without damaging the mechanical and aesthetic performance. Besides, it has low cost compared to indirect materials. [4,12-14]

This material consists of an organic matrix of polymerizable resin bonded chemically to filler particles by the bonding agent, silane [15]. Nanocomposite was the restorative material used in both cases since their indication is already proved in the literature for restorations of anterior teeth. Nanocomposite contains filler particles no larger than $0.1 \mu$ (more generally $0.04-0.05 \mu$ ) so there are some advantages in comparison to others resins such as good physical and optical properties besides the excellent polish [2].

The restorative treatments involve significant modifications in both occlusion and aesthetic parameters. Thus, mock-up is widely used during the planning of clinical procedures, since it is possible to evaluate and control, for example, the thickness of the dental preparations as well as the volume of the final restorations [16]. Additionally, clinical procedures that do not require tooth structure wear, do not compromise its integrity or functionality, but, with the addition of restorative material, minor modifications may occur in its functionality. Therefore, esthetic rehabilitation should include reestablishment of occlusion, such as anterior and lateral guidance as shown in cases 1 and 2 . Besides, not only the esthetic design is important, but also the new emergency profile to improve the incisal reestablishment with the lips and the anterior and lateral disocclusion guides [17].

The failure in anterior restorations are related more to esthetic appearance such as color alterations, surface staining and marginal mismatch than secondary caries that is observed in posterior restorations [2,18]. In a study, anterior composite restorations placed by general dental practitioners showed an annual failure rate of $4.9 \%$, with relevant differences among practitioners [19]. In another study, between three and nine years of follow-up the annual failure rates (AFRs) for anterior composite resin restorations were 0 to $3.7 \%$, while for those studies with over 10 years of follow-up the AFRs were between $0.6 \%$ and $4.1 \%$. This could be an indication for an increasing failure rate over time, which is explained by the increase of marginal staining and discoloration reducing esthetical appearance [18].

Rubber dam isolation is still a "gold standard" approach to keep the operatory field dry and free of contaminants like saliva for restorative procedures [10].

To have a good esthetic predictability performance and save chair time in the performance of restorations it is also indicated the use of the lingual matrix technique that provides the clinician with a palatal guide to develop the correct lingual proportion and shape created in the diagnostic waxing [6].

For anterior teeth restorations with composite resin, the most indicated technique is the layering technique. The advantages of this technique are better light-curing of each resin increment and low cavity configuration factor, since the volume of contracting material is reduced, avoiding polymerization shrinkage stresses [2].

The main disadvantage of the composite resin is the staining or discoloration of the restorative material after aging process in the oral environment induced by several extrinsic or intrinsic factors $[20,21]$. Therefore, the surface quality of restorations is considered a key factor for clinical success [22].

Smooth and polished restorations increase the lifetime of composite resin restorations, are less susceptible to plaque accumulation and surface pigmentation and optimizes their esthetic appearance. In this context, the finishing and polishing techniques used are important steps to enhance both the esthetics and longevity $[2,22]$. According to Chour et al, diamond polishing paste can be used to remove rough surface whereas sof-lex for final finish and polish of the composite restoration, increasing longevity of restorations [23]. 
The importance of study the case with the help of dental photography and diagnostic waxing for posterior fabrication of the lingual matrix guide were reinforced to simplify the technique and have predictable long-term results.

\section{CONCLUSION}

To conclude, the tooth recontouring techniques and the replacement of extensive restorations with composite resin restorations provide an ultra-minimally invasive protocol with excellent esthetic appearances. The resin composite restorations can promote an effective long-term performance if the correct technique is performed, a proper treatment plan and periodic controls.

\section{REFERENCES}

1. A. Mathias P, da Silva EV, Aguiar TR, Andrade AS, Azevedo J. A Conservative Esthetic Approach Using Enamel Recontouring and Composite Resin Restorations. Case Rep Dent. 2016;2016:1254610. Epub 2016 0ct 12.

2. Gouveia THN, Theobaldo JD, Vieira-Junior WF. Esthetic smile rehabilitation of anterior teeth by treatment with biomimetic restorative materials: a case report. Clin Cosmet Investig Dent. 2017 May 11;9:27-31.

3. Frese C, Schiller P, Staehle HJ, Wolff D. Recontouring teeth and closing diastemas with direct composite buildups: a 5-year follow-up. J Dent. 2013 Nov;41(11):979-85.

4. Coelho-de-Souza FH, Gonçalves DS, Sales MP, Erhardt MC, Corrêa MB, Opdam NJ, et al. Direct anterior composite veneers in vital and non-vital teeth: a retrospective clinical evaluation. J Dent. 2015 Nov;43(11):1330-6.

5. Staehle HJ, Wolff D, Frese C. More conservative dentistry: clinical long-term results of direct composite resin restorations. Quintessence Int. 2015 May;46(5):373-80

6. Romero MF.Esthetic anterior composite resin restorations using a single shade: Step-by-step technique. J Prosthet Dent. 2015 Jul;114(1):9-12.

7. Demirci M, Tuncer S, Özta E, Tekçe N, Uysal Ö. A 4-year clinical evaluation of direct composite build-ups for space closure after orthodontic treatment. Clin Oral Investig. 2015 Dec;19(9):2187-99.

8. Heintze SD, Rousson V, Hickel R. Clinical effectiveness of direct anterior restorations-A meta-analysis. Dent Mater. 2015 May;31(5):481-95.

9. Rosa WL, Piva E, Silva AF. Bond strength of universal adhesives: $A$ systematic review and meta-analysis. J Dent. 2015 Jul;:43(7):765-76.
10. Báez Rosales A, De Nordenflycht Carvacho D, Schlieper Cacciutolo R, Gajardo Guineo M, Gandarillas Fuentes C. Conservative Approach for the Esthetic Management of Multiple Interdental Spaces: A Systematic Approach. J Esthet Restor Dent. 2015 Nov-Dec;27(6):344-54.

11. Demarco FF, Collares K, Correa MB, Cenci MS, Moraes RR, Opdam NJ. Should my composite restorations last forever? Why are they failing? Braz Oral Res. 2017 Aug 28;31(suppl 1):e-56.

12. Campos RE, Miranda Valdivia AD, Santos-Filho PC, Menezes Mde S, de Oliveira Junior OB, Soares CJ. Conservative treatment for amelogenesis imperfecta: a case report. Gen Dent. 2014 Jan-Feb;62(1):74-8.

13. Vadini M, D'Amario M, De Angelis F,Falco A, D'Arcangelo C. No-Prep Rehabilitation of Fractured Maxillary Incisors with Partial Veneers. J Esthet Restor Dent. 2016 Nov 12;28(6):351-8.

14. da Cunha LF, Gaião U, Silva RC, Gonzaga CC, Correr GM. Cosmetic Remodeling of the Smile: Combining Composite Resin and Ceramics over Teeth and Implants. Case Rep Dent. 2017;2017:8698010. doi: 10.1155/2017/8698010. Epub 2017 Aug 3.

15. Moda MD, Godas AGL, Fernandes JC, Suzuki TYU, Guedes APA, Briso ALF, et al. Comparison of different polishing methods on the surface roughness of microhybrid, microfill, and nanofill composite resins. J Investig Clin Dent. 2018 Feb;9(1). doi: 10.1111/jicd.12287. Epub 2017 Aug 1.

16. Fabbri G, Cannistraro G, Pulcini C, Sorrentino R. The full-mouth mock-up: a dynamic diagnostic approach (DDA) to test function and esthetics in complex rehabilitations with increased vertical dimension of occlusion. Int J Esthet Dent. 2018;13(4):460-74.

17. da Cunha LF, Prochnow RA, Costacurta A0, Gonzaga CC, Correr GM. Replacement of anterior composite resin restorations using conservative ceramics for occlusal and periodontal rehabilitation: an 18-month clinical follow-up. Case Rep Dent. 2016;2016:9728593. doi: 10.1155/2016/9728593. Epub 2016 Jul 31.

18. Demarco FF, Collares K, Coelho-de-Souza FH, Correa MB, Cenci MS, Moraes RR, et al. Anterior composite restorations: A systematic review on long-term survival and reasons for failure. Dent Mater.2015 0ct;31(10):1214-24. doi: 10.1016/j.dental.2015.07.005. Epub 2015 Aug 21.

19. Collares K, Opdam NJM, Laske M, BronkhorstEM, Demarco FF, Correa MB, et al. Longevity of Anterior Composite Restorations in a General Dental Practice-Based Network. J Dent Res. 2017 Sep;96(10):1092-9.

20. Özdaş DÖ, Kazak M, Çilingir A, Subaşı MG, Tiryaki M, Günal Ş. Color Stability of Composites After Short-term Oral Simulation: An in vitro Study. Open Dent J. 2016 Aug 31;10:431-7.

21. Poggio C, Vialba L, Berardengo A, Federico R, Colombo M, Beltrami R, et al. Color Stability of New Esthetic Restorative Materials: A Spectrophotometric Analysis. J Funct Biomater.2017 Jul 6;8(3). pii: E26. doi: 10.3390/jfb8030026.

22. Carneiro P, Ramos TM, de Azevedo CS, de Lima E, de Souza S, Turbino ML, et al. Influence of Finishing and Polishing Techniques and Abrasion on Transmittance and Roughness of Composite Resins. Oper Dent. 2016 Nov/ Dec;41(6):634-41.

23. Chour RG, Moda A, Arora A, Arafath MY, Shetty VK, Rishal Y. Comparative evaluation; of effect of different polishing systems on surface roughness of composite resin: an in vitro study. J Int Soc Prev Community Dent. 2016 Aug;6(Suppl 2):S166-70. doi: 10.4103/2231-0762.189761.

\section{Priscila Regis Pedreira} (Corresponding address)

Piracicaba Dental School, University of Campinas, Av. Limeira, 901, P.O. BOX 52

Piracicaba, SP, Brazil, CEP 13414-903.

Telephone: +55 71 99242-9972,

E-mail: priscilaregis1@hotmail.com

Date submitted: 2018 Aug 01 Accept submission: 2018 Dec 18 